\title{
SpaceOps 2012 Plus 2: Social Tools to Simplify ISS Flight Control Communications and Log Keeping
}

\author{
Hugh S. Cowart ${ }^{1}$ and David W. Scott. ${ }^{2}$ \\ NASA - Marshall Space Flight Center, Huntsville, AL, 35812, USA
}

\begin{abstract}
A paper written for the SpaceOps 2012 Conference (Simplify ISS Flight Control Communications and Log Keeping via Social Tools and Techniques) identified three innovative concepts for real time flight control communications tools based on social mechanisms: a) Console Log Tool (CoLT) - A log keeping application at Marshall Space Flight Center's (MSFC) Payload Operations Integration Center (POIC) that provides "anywhere" access, comment and notifications features similar to those found in Social Networking Systems (SNS), b) Cross-Log Communication via Social Techniques - A concept from Johnsson Space Center's (JSC) Mission Control Center Houston (MCC-H) that would use microblogging's @tag and \#tag protocols to make information/requests visible and/or discoverable in logs owned by@Destination addressees, and c) Communications Dashboard (CommDash) - A MSFC concept for a Facebook-like interface to visually integrate and manage basic console log content, text chat streams analogous to voice loops, text chat streams dedicated to particular conversations, generic and position-specific status displays/streams, and a graphically based hailing display. CoLT was deployed operationally at nearly the same time as SpaceOps 2012, the CrossLog Communications idea is currently waiting for a champion to carry it forward, and CommDash was approved as a NASA Iinformation Technoloby (IT) Labs project. This paper discusses lessons learned from two years of actual CoLT operations, updates CommDash prototype development status, and discusses potential for using Cross-Log Communications in both MCC-H and/or POIC environments, and considers other ways for synergizing console applcations.
\end{abstract}

\footnotetext{
${ }^{1}$ Data Management Coordinator, Mission Operations Laboratory, MSFC/EO30, hugh.s.cowart@nasa.gov

${ }^{2}$ Computer Engineer, Ground Systems Development \& Integration, MSFC/EO50, scotty@nasa.gov 